\title{
Advanced CT acquisition protocol with a third-generation dual-source CT scanner and iterative reconstruction technique for comprehensive prosthetic heart valve assessment
}

\author{
Marguerite E. Faure ${ }^{1,2} \cdot$ Laurens E. Swart ${ }^{2,3}$ - Marcel L. Dijkshoorn ${ }^{2}$. Jos A. Bekkers ${ }^{4}$. \\ Marcel van Straten ${ }^{2}$ - Koen Nieman ${ }^{3}$ • Paul M. Parizel ${ }^{1}$ • Gabriel P. Krestin ${ }^{2}$. \\ Ricardo P. J. Budde ${ }^{2}$
}

Received: 17 July 2017 / Revised: 19 October 2017 / Accepted: 30 October 2017 /Published online: 12 December 2017

(C) The Author(s) 2017. This article is an open access publication

\begin{abstract}
Objectives Multidetector CT (MDCT) is a valuable tool for functional prosthetic heart valve (PHV) assessment. However, radiation exposure remains a concern. We assessed a novel CT-acquisition protocol for comprehensive PHV evaluation at limited dose.

Methods Patients with a PHV were scanned using a thirdgeneration dual-source CT scanner (DSCT) and iterative reconstruction technique (IR). Three acquisitions were obtained: a non-enhanced scan; a contrast-enhanced, ECG-triggered, arterial CT angiography (CTA) scan with reconstructions at each $5 \%$ of the R-R interval; and a delayed high-pitch CTA of the entire chest. Image quality was scored on a five-point scale. Radiation dose was obtained from the reported CT dose index (CTDI) and dose length product (DLP).

Results We analysed $43 \mathrm{CT}$ examinations. Mean image quality score was $4.1 \pm 1.4,4.7 \pm 0.5$ and $4.2 \pm 0.6$ for the non-contrastenhanced, arterial and delayed acquisitions, respectively, with a total mean image quality of $4.3 \pm 0.7$. Mean image quality for leaflet motion was 3.9 \pm 1.4 . Mean DLP was $28.2 \pm 17.1,457.3$ \pm 168.6 and $68.5 \pm 47.2 \mathrm{mGy} . \mathrm{cm}$ for the non-contrast-enhanced
\end{abstract}

Marguerite E. Faure

marguerite.faure@uza.be

Department of Radiology, University Hospital of Antwerp, Wilrijkstraat, 102650 Edegem, Belgium

2 Department of Radiology \& Nuclear Medicine, Erasmus MC, Rotterdam, The Netherlands

3 Department of Cardiology, Erasmus MC, Rotterdam, The Netherlands

4 Department of Cardiothoracic surgery, Erasmus MC, Rotterdam, The Netherlands $(n=40)$, arterial $(n=43)$ and delayed acquisition $(n=43)$, respectively. The mean total DLP was $569 \pm 208 \mathrm{mGy} . \mathrm{cm}$ and mean total radiation dose was $8.3 \pm 3.0 \mathrm{mSv}(\mathrm{n}=43)$.

Conclusion Comprehensive assessment of PHVs is possible using DSCT and IR at moderate radiation dose.

Key points

- Prosthetic heart valve dysfunction is a potentially lifethreatening condition.

- Dual-source CT can adequately assess valve leaflet motion and anatomy.

- We assessed a comprehensive protocol with three acquisitions for PHV evaluation.

- This protocol is associated with good image quality and limited dose.

Keywords Prosthetic heart valve · Image quality $\cdot$ Cardiac imaging $\cdot$ Computed tomography $\cdot$ Radiation dose

$\begin{array}{ll}\text { Abbreviations } \\ \text { CTA } & \text { Computed tomography angiography } \\ \text { DSCT } & \text { Dual-source computed tomography } \\ \text { IR } & \text { Iterative reconstruction } \\ \text { MDCT } & \text { Multidetector computed tomography } \\ \text { PHV } & \text { Prosthetic heart valve } \\ \text { PVL } & \text { Paravalvular leakage } \\ \text { TEE } & \text { Transoesophageal echocardiography } \\ \text { TTE } & \text { Transthoracic echocardiography }\end{array}$

\section{Introduction}

Prosthetic heart valve (PHV) dysfunction is an uncommon but important and potentially life-threatening complication 
after PHV implantation [1]. In daily practice, the most commonly used imaging modality for evaluating PHVs is transthoracic echocardiography (TTE) [2]. TTE has known limitations such as a high interobserver variability, the possibility of poor acoustic windows and acoustic shadowing. Hence, evaluation of PHV abnormalities often also requires transoesophageal echocardiography (TEE), which is a semi-invasive procedure associated with certain risks and complications, and is also affected by some of the TTE limitations, albeit to a lesser extent [3].

More recently, multidetector computed tomography (MDCT) has been shown to provide complementary information for PHV assessment and is useful to identify the cause of PHV dysfunction, including obstructive masses (such as pannus and thrombus), prosthesis-patient mismatch, paravalvular regurgitation, as well as infective endocarditis and its complications $[4,5]$. Most often, a retrospectively ECG-gated CT angiography (CTA) acquisition is used to assess PHV dysfunction since it allows for reconstructions at each $5-10 \%$ of the R-R interval needed to dynamically assess valve leaflet motion and anatomy. Such acquisitions are associated with a relatively high radiation dose as reported in multiple studies (mean $11.6 \mathrm{mSV}$ [interquartile range (IQR) 10.8-14.4], $11.8 \mathrm{mSV}$ [IQR 11.2-12.8] and $18.8 \pm 3.8 \mathrm{mSV}$ [IQR 6, 7, 8]). Ideally, a CT acquisition protocol for PHV assessment would not only include a dynamic CTA but also a non-enhanced scan of the valve region to assess calcifications and suture pledgets, as well as a delayed phase of the entire chest for possible abscesses, septic pulmonary emboli and overall thoracic anatomy, which would increase radiation dose even further [9]. Third-generation dual-source scanners allow for several dose-reduction strategies that are advantageous for PHV assessment. We examined whether a novel, moderate radiation dose three-phase CT acquisition protocol for thirdgeneration dual-source CT (DSCT) allows comprehensive ECG-triggered PHV assessment with both static and dynamic reconstructions to assess the PHV region as well as the entire chest.

\section{Materials and methods}

\section{Patients}

All consecutive patients with a PHV that underwent a CT can with this specific acquisition protocol in our department between December 2014 and November 2016 were included. The acquisition was a part of the routine clinical workup, and data were gathered retrospectively. No additional acquisitions were made specifically for this study. Patient data were retrieved from the electronic patient files. A waiver for retrospective evaluation of the data was obtained from the medical ethics committee.

\section{CT scan protocol}

Image acquisition was performed on a third-generation DSCT (SOMATOM Force, Siemens, Erlangen, Germany). Image acquisition included three consecutive scans (Table 1, Fig. 1).

First, a non-enhanced prospectively ECG-triggered scan of the PHV region alone was performed with the following acquisition parameters: fixed tube voltage $120 \mathrm{kV}$, reference effective tube load $80 \mathrm{mAs}$, collimation adapted to fit scan length, and gantry rotation time $250 \mathrm{~ms}$. Data were acquired and reconstructed (slice thickness $3 \mathrm{~mm}$, increment $3 \mathrm{~mm}$, Qr36 kernel) for the $45 \%$ phase of the R-R interval with iterative reconstruction (IR) (Admire Level 3, Siemens).

Secondly, a prospectively ECG-triggered wide-pulsing window sequential CT angiography (CTA) was performed with the following parameters: fixed tube voltage of $120 \mathrm{kV}$, reference effective tube load $180 \mathrm{mAs}$, collimation $192 \times$ $0.6 \mathrm{~mm}$ and gantry rotation time of $250 \mathrm{~ms}$. This prospectively ECG-triggered sequential scan was made with three, five or seven stacks. Care was taken to centre the middle stack on the prosthetic valve to avoid any stack artefacts at the level of the valve. For three stacks, the scan length in the z-axis was set at $14 \mathrm{~cm}$, to allow for a maximum coverage per stack in the longitudinal direction (Figs. 2 and 3). Reducing the scan length will reduce the coverage of each of the three stacks evenly until the coverage can be accommodated by two stacks, thereby increasing the risk of stack artefacts. For this CTA, we used an ECG-trigger based on an absolute delay time after an R-peak instead of a relative (\%) R-R trigger delay. Irrespective of heart rate, a fixed 'scan-on-scan-off' padding setting from $0-1,500 \mathrm{~ms}$ with $50-500 \mathrm{~ms}$ ECG-pulsing was used, which resulted in a scan acquisition window that is guaranteed to provide the fastest onset of radiation after an R-peak. After $500 \mathrm{~ms}$, tube current (and thus radiation) decreased from $100 \%$ to $20 \%$ of the set level. Radiation was then maintained for $1,500 \mathrm{~ms}$ (which is a full heart cycle at 40 $\mathrm{bpm}$ ) or cut off when the next R-peak occurred. Reconstructions were made at each $5 \%$ of the R-R interval, with a slice thickness of $0.75 \mathrm{~mm}$, an increment of $0.4 \mathrm{~mm}$ and Bv40 kernel (Figs. 2 and 3). An iterative reconstruction technique was used (Admire level 3, Siemens).

Thirdly and finally, a low-dose high-pitch CTA of the entire chest was performed, which was triggered in such a way that the scan reaches the trachial carina level at $30 \%$ of the R-R interval. The scan was made with the following parameters: automatically selected tube voltage, reference effective tube load $180 \mathrm{mAs}$ at $120 \mathrm{kV}$, collimation $192 \times 0.6 \mathrm{~mm}$, and a pitch of 3.2. Reconstructions were made with an increment of $0.4 \mathrm{~mm}$, a slice thickness of $0.75 \mathrm{~mm}$ in Bv40 kernel and iterative reconstruction (Admire level 3, Siemens). 
Table 1 Prosthetic heart valve scan protocol

\begin{tabular}{|c|c|c|c|}
\hline Prosthetic valve protocol & Non-enhanced scan & Contrast-enhanced, ECG-triggered, CTA & Delayed high-pitch CTA of the entire chest \\
\hline Scan start & $2 \mathrm{~cm}$ above valve & Center of scan centered & $2 \mathrm{~cm}$ above arch \\
\hline Scan end & $2 \mathrm{~cm}$ below valve & On valve & Bottom of heart \\
\hline Scan length & Variable & Fixed 14 cm (3 stacks) & Variable \\
\hline Scan type & Prospective step and shoot & Prospective step and shoot & Prospective high pitch spiral flash \\
\hline Collimation & Adapted to scan length & $192 \times 0.6 \mathrm{~mm}$ & $192 \times 0.6 \mathrm{~mm}$ \\
\hline Rotation time (ms) & 250 & 250 & 250 \\
\hline Pitch & n.a. & n.a. & 3.2 \\
\hline Feed/rot $(\mathrm{mm})$ & variable & 48 & 184 \\
\hline Reference mAs/rot & 80 & 180 & 180 \\
\hline mA modulation & Yes, CARE DOSE 4D & Yes, CARE DOSE 4D & Yes, CARE DOSE 4D \\
\hline Reference $\mathrm{kVp}$ & 120 & 120 & 120 \\
\hline $\mathrm{kVp}$ modulation & CARE kV semi 120 & CARE kV semi 120 & CARE kV semi 120 \\
\hline Tissue of interest & Non-contrast & Vascular & Vascular \\
\hline Beta blocker/Nitrates & no & no & no \\
\hline ECG padding & $45 \%-45 \%$ & $0-1,500 \mathrm{~ms}$ & n.a. \\
\hline ECG pulsing & off & $50-500 \mathrm{~ms}$ & n.a. \\
\hline Contrast & no & yes & yes \\
\hline Type & & Iodixanol*320mgl $/ \mathrm{ml}$ & \\
\hline Bolus & & $80 \mathrm{ml}$ at $5 \mathrm{ml} / \mathrm{s}$ & \\
\hline Chaser (30\% mix ratio) & & $20 \mathrm{ml}$ at $3.5 \mathrm{ml} / \mathrm{s}$ & \\
\hline delay & & Bolustracking & 70 s post-injection \\
\hline Slice width $(\mathrm{mm})$ & 3.0 & 0.75 & 0.75 \\
\hline Slice increment (mm) & 3.0 & 0.4 & 0.4 \\
\hline Kernel & Qr36 & Bv40 & Bv40 \\
\hline ADMIRE strength & 3 & 3 & 3 \\
\hline ECG phase \% & $45 \%$ & $10-100 \%$ at $5 \%$ interval & $30 \%$ at trachial carina level \\
\hline ECG phase ms & n.a. & Best systolic ms phase by computer & n.a. \\
\hline
\end{tabular}

*Visipaque, GE Healthcare

CTA CT angiography

A single biphasic contrast administration protocol was used in which a total amount of $100 \mathrm{ml}$ of contrast was given with an Iodine concentration of $320 \mathrm{mg} / \mathrm{ml}$ (Visipaque 320®, GE Healthcare, Cork, Ireland). First, $80 \mathrm{ml}$ of contrast was injected at a rate of $5.0 \mathrm{ml} / \mathrm{s}$, followed by $20 \mathrm{ml}$ of contrast at $3.5 \mathrm{ml} / \mathrm{s}$ and a saline flush of $25 \mathrm{ml}$ at $2.5 \mathrm{ml} / \mathrm{s}$. A region of interest was defined in the ascending aorta and data acquisition was initialized when the threshold of $100 \mathrm{HU}$ was reached. The delayed acquisition was obtained $70 \mathrm{~s}$ after contrast injection. No beta blockers or nitroglycerin were administered since they may be contraindicated in patients with valvular pathology.

Radiation dose was obtained from the reported CT dose index (CTDI) and dose length product (DLP) and converted to an effective dose, using a conversion factor of $0.0145 \mathrm{mSv} /$ $\mathrm{mGy}^{*} \mathrm{~cm}[10]$. Heart rate during the acquisitions was obtained from the automatically generated information stored with the DICOM images.

\section{Image analysis}

Assessment of the CT examinations was performed on a dedicated workstation (Philips Intellispace Portal, v6.0.3.12200). Using multiplanar reformation, images were reconstructed in plane with the PHV and perpendicular to the PHV and valve leaflets. Cine mode was used to dynamically evaluate PHV leaflet motion in all reconstructed phases of the RR interval. Image quality was scored separately for each of the three acquisitions in the same way, using a 5-point scale, adapted from a 4-point scoring system used in a previous study by our group [11]. The criteria for the different scores were formulated as follows: 1 - no discernible detail, no diagnostic information can be obtained; 2 - only limited visualization of the region of interest, no accurate measurements can be made; 3 image quality is moderate, measurements and diagnosis are possible; 4 - image quality is good, no diagnostic or 
Fig. 1 Image acquisition includes three sequential acquisitions: A non-contrastenhanced scan (A); a contrastenhanced arterial CT angiography (CTA) with reconstructions at each $5 \%$ of the R-R interval (B); and a delayed high pitch CTA of the entire chest $(\mathbf{C})$, which allows for evaluation of the complete thoracic aorta as shown on this sagittal view (D)
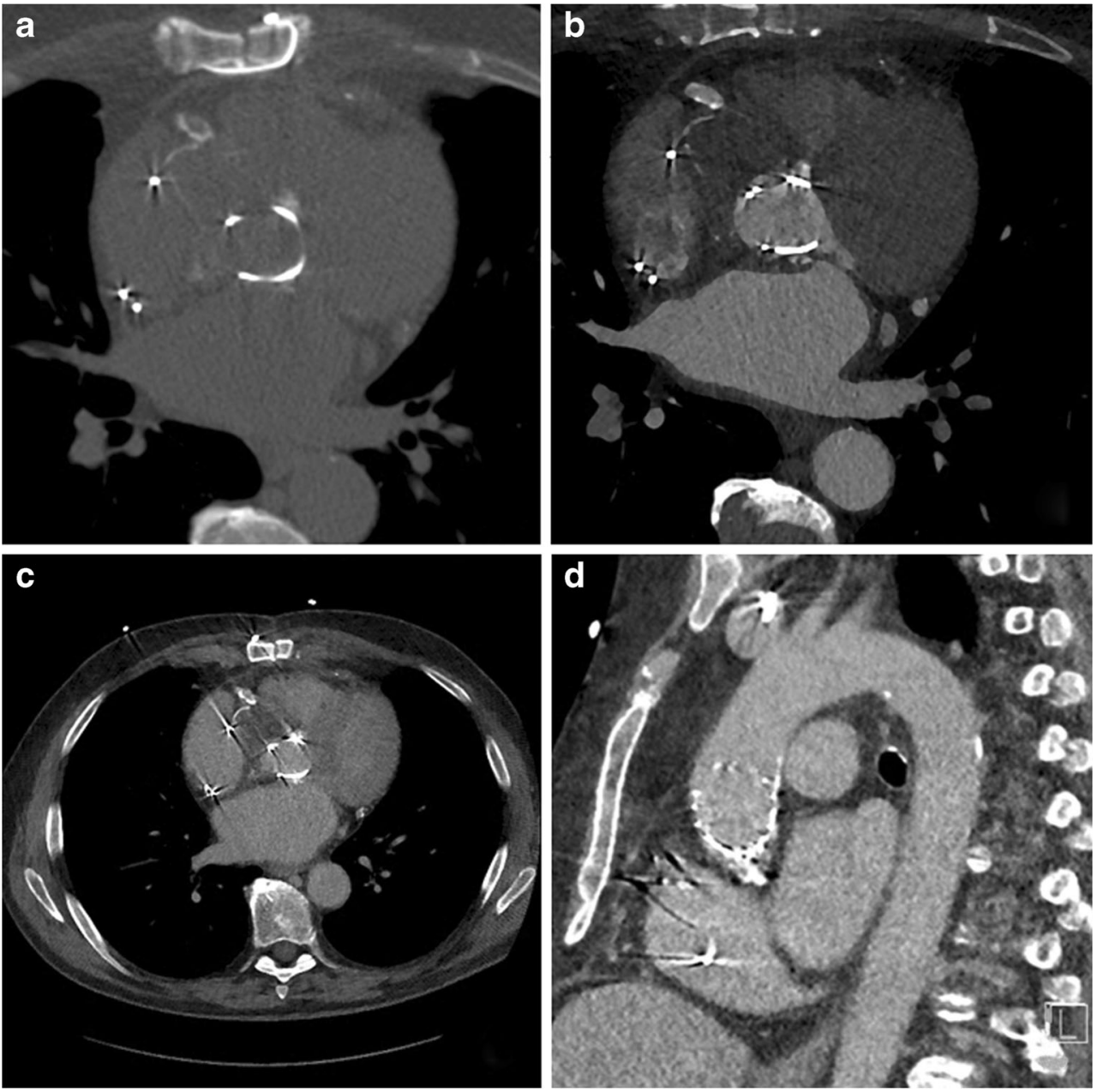

measurement problems; 5 - image quality is excellent. Furthermore, image quality of PHV leaflet motion was scored in the same way: 1 - leaflet motion not assessable; 2 - leaflet motion assessable, no reliable measurements can be made; 3 leaflet motion assessable with the ability to measure opening and closing angles; 4 - good image quality of the leaflet

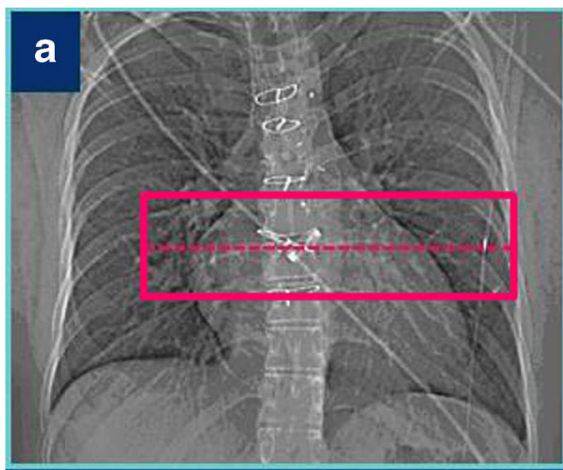

Fig. 2 CT angiography (CTA) acquisition: Effect of scan range length in CTA: With prospective protocols in third-generation dual-source CTA the collimation is adapted to the set scan length. In regular cardiac examinations, this helps to avoid overscanning and results in an interpatient variability of the coverage per stack. When scanning heart valves full heart coverage is not always necessary and a shorter range may be sufficient. Special care should be taken in planning the scan range, however, so the largest possible $z$ coverage per stack is obtained to avoid

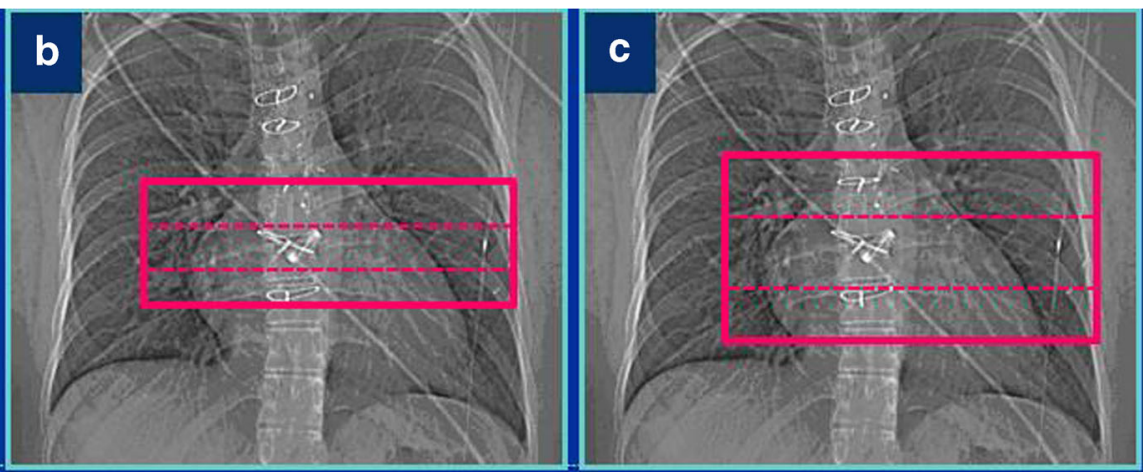

stack artefacts at the valve level. A A short scan range consisting of two stacks will result in potential stack artefacts through the valve. B A slightly longer scan range will result in three thin collimate stacks with the valve in the middle of the mid stack. Heart motion and respiratory variability might still move the valve into the potential stack artefacts. $\mathbf{C}$ Opening the scan length to exactly $14 \mathrm{~cm}$ will result in three stacks with maximum stack coverage ensuring valve images without ECG stack artefacts 


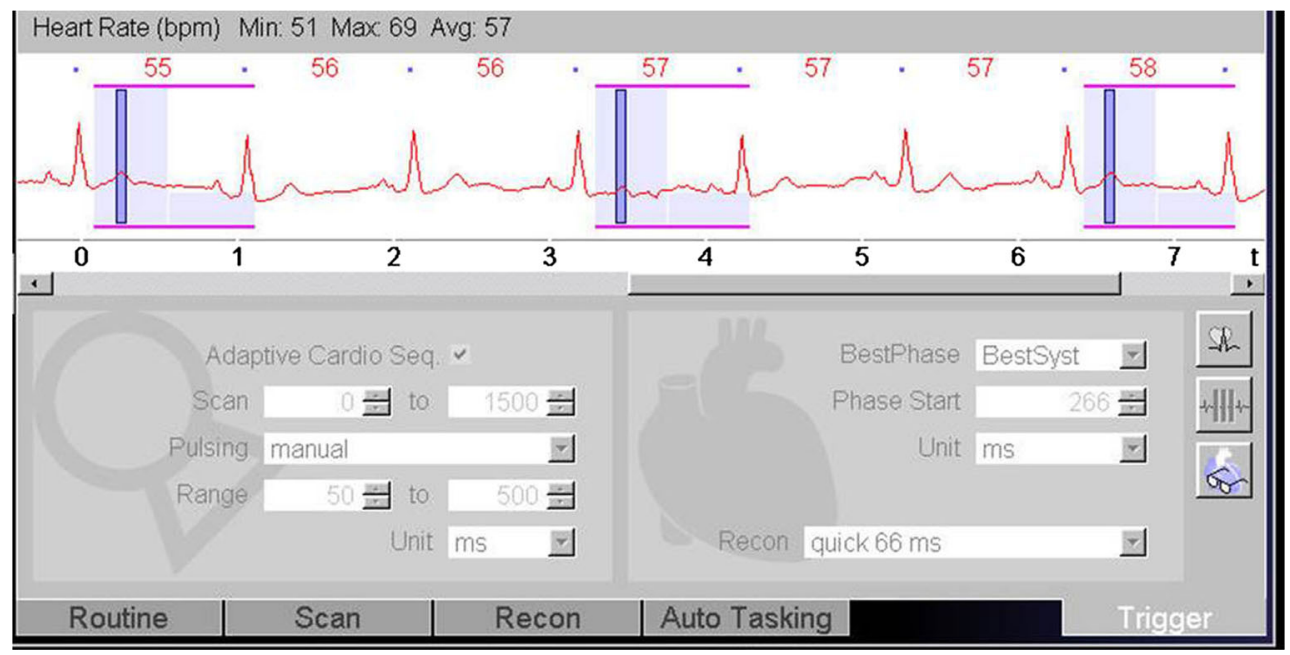

Fig. 3 Screenshot from scanner console: The ECG-trigger in this CTA acquisition is based on absolute millisecond ( $\mathrm{ms}$ ) time instead of a relative (\%) RR-trigger. A 'scan-on-scan-off' padding setting from 0-1,500 ms with $50-500 \mathrm{~ms}$ ECG-pulsing results in a scan acquisition window that is guaranteed to provide the fastest onset of radiation after an R-peak (minimum delay is $\pm 100 \mathrm{~ms}$, which is $10 \%$ at $60 \mathrm{bpm}$ ). Radiation is maintained for $1,500 \mathrm{~ms}$ (which is a full heart cycle at $40 \mathrm{bpm}$ ) or is cut off when the next R-peak occurs. This means that independent of the heart rate, a full heart cycle (starting from $100 \mathrm{~ms}$ ) is guaranteed

increased pressure gradient or suspicion of valve obstruction $(n=10)$, suspicion or follow-up of paravalvular leakage $(n=4)$. Other indications were follow-up after coarctation repair $(n=2)$, after tetralogy of Fallot repair $(n=1)$, follow-up of Marfan's disease $(n=1)$, after a Bentall procedure $(n=1)$, after closure of a pseudo-aneurysm $(\mathrm{n}=1)$ and newly diagnosed pleural effusion $(\mathrm{n}=1)$.

\section{CT parameters}

The non-enhanced acquisition was not performed in three patients. In all other patients $(n=40)$, the three acquisitions were obtained. Mean heart rate during scanning was $70 \pm 19 \mathrm{bpm}$ for the non-enhanced scan, $69 \pm 13 \mathrm{bpm}$ for the ECG-triggered CTA and $75 \pm 25 \mathrm{bpm}$ for the high-pitch CTA. Heart rate variability was calculated for the different stacks of the noncontrast-enhanced scan and the CTA, using 1 standard deviation of the mean heart rate. This was $2.3 \mathrm{bpm}$ for the noncontrast-enhanced scan and $2.8 \mathrm{bpm}$ for the CTA. The maximum heart rate variability was $24 \mathrm{bpm}$ for the non-enhanced scan and $32.3 \mathrm{bpm}$ for the CTA. The number of stacks used was $1(n=10), 2(n=23), 3(n=5)$ and $4(n=2)$ for the nonenhanced scan and $3(n=41), 5(n=1)$ and $7(n=1)$ for the ECG-triggered CTA. Stack artefacts were seen in one out of 30 non-contrast enhanced CT scans (ten non-contrast scans were scanned with only one stack, so artefacts could not occur) and in two out of 43 ECG-triggered CTAs. In all these examinations, the artefacts did not appear at the valve position. In the delayed acquisition with automatic tube voltage selection, in one of 43 scans ( $2 \%$ ) the reference voltage of $120 \mathrm{kV}$ was chosen, in three scans (7\%) the tube voltage was 
Table 2 Patient characteristics

\begin{tabular}{llll}
\hline Gender $(\mathrm{n}=41)$ & 22 male & 19 female & \\
\hline Age, years (mean) $(\mathrm{n}=41)$ & $60 \pm 23$ & & \\
PHV position (n=44) & Aortic: 35 & Mitral: 6 & Pulmonary: 3 \\
PHV type (n=44) & Mechanical: 21 & Biological: 12 & TAVI: 11 \\
& St-Jude: 19 & Perimount: 6 & Corevalve: 6 \\
& Carbomedics: 1 & Sorin: 1 & Lotus: 3 \\
& Björk-Shiley: 1 & Perigon: 1 & Sapien: 2 \\
& & Homograft: 4 & \\
\hline
\end{tabular}

PHV prosthetic heart valve

$100 \mathrm{kV}$, in four scans $(9 \%) 70 \mathrm{kV}$, in nine scans $(21 \%) 90 \mathrm{kV}$ and in most of the scans (26 scans, $61 \%$ ) $80 \mathrm{kV}$ was chosen.

\section{Radiation dose}

Mean CTDI was 3.13 $\pm 1.28,30.92 \pm 9.6$ and $2.31 \pm 0.94 \mathrm{mGy}$ for the non-enhanced $(n=40)$, CTA $(n=43)$ and delayed acquisitions ( $n=43$ ), respectively. Mean DLP was 28.2 $\pm 17.1,457.3$ \pm 168.6 and $68.5 \pm 47.2 \mathrm{mGy} . \mathrm{cm}$ for the non-contrast-enhanced $(n=40)$, arterial $(n=43)$ and delayed acquisition $(n=43)$, respectively. The mean total DLP was $569 \pm 208 \mathrm{mGy} . \mathrm{cm}$. Mean total radiation dose was $8.3 \pm 3.0 \mathrm{mSv}$ (range 3-17, $\mathrm{n}=43$ ) (Table 3 ). In one study, the arterial CTA had seven stacks, with a wider scan range due to coronary artery bypass grafting $(\mathrm{CABG})$, which resulted in a total dose of $16.7 \mathrm{mSv}$. In two studies, the abdomen was included in the high-pitch acquisition with a total radiation dose of $16.8 \mathrm{mSv}$ and 6.7 $\mathrm{mSv}$, respectively. Furthermore, one study had an additional high-pitch acquisition with a total radiation dose of $12.7 \mathrm{mSv}$.

\section{Image quality scores}

The mean image quality score was $4.1 \pm 1.4,4.7 \pm 0.5$ and 4.2 \pm 0.6 for the non-enhanced, arterial CTA and delayed acquisition, respectively, with a total mean image quality of $4.3 \pm 0.7$

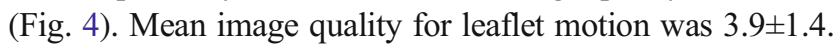
Only one non-enhanced scan had a score of 1 and was not interpretable due to the presence of a cobalt- and chromiumcontaining mechanical heart valve (Björk-Shiley valve), which gives substantial artefacts. Another non-enhanced scan had a score of 2 with limited image quality due to a too short scan length with incomplete visualization of the heart. Overall, 124 out of 126 (98\%) acquisitions (40 non-contrast-enhanced, 43 CTA and 43 delayed acquisitions) had an image quality score of $\geq 3$. Regarding leaflet motion, four scans had a score of 1 and were non-diagnostic and four had a score of 2 with only poor image quality. These were five transcatheter valves (four Corevalves and one Sapien valve) and three conventional biological valves. Thirty-four scans had an image quality score of $\geq 3(81 \%)$ with respect to leaflet motion.

Out of 43 examinations, 16 showed no PHV-related abnormalities. Five examinations revealed a false aneurysm at the aortic root. Three examinations showed a clear paravalvular leakage (PVL) and four had possible PVL (Fig. 5). Five examinations showed pannus or thrombus under the PHV and six examinations showed vegetations/structures inside the PHV. Abscess formation was found in one examination. Five examinations showed a cavity under the PHV. Three examinations revealed thrombus in the left atrial appendage. On the other hand, two patients had suboptimal filling of the left atrial appendage at the CTA, but thrombus could be excluded with the delayed scan.

\section{Interobserver agreement}

The weighted kappa value for the image quality scores of both observers was 0.74 for the non-contrast-enhanced scan, 0.87 for the CTA and 0.80 for the delayed high-pitch scan. For scoring image quality of leaflet motion the kappa value was
Table 3 Radiation dose overview of the different acquisitions

\begin{tabular}{lccc}
\hline & Non-contrast CT scan $($ mean \pm SD) & $\begin{array}{c}\text { Arterial CT scan } \\
(\text { mean } \pm \text { SD) }\end{array}$ & Delayed CT scan (mean \pm SD) \\
\hline CTDI (mGy) & $3.13 \pm 1.27$ & $31.01 \pm 9.57$ & $2.32 \pm 0.93$ \\
DLP (mGycm) & $28.1 \pm 16.9$ & $458.3 \pm 166.7$ & $68.7 \pm 46.7$ \\
Dose (mSv) & $0.41 \pm 0.23$ & $6.65 \pm 2.42$ & $1.00 \pm 0.68$ \\
\hline
\end{tabular}

CTDI CT dose index, DLP dose length product 
Fig. 4 Image quality. Example of $\mathrm{CT}$ angiography (CTA)

reconstruction in systole and diastole showing excellent image quality of a bi-leaflet prosthetic heart valve in the aortic position with valve in open $(\mathbf{A})$ and closed (B) position
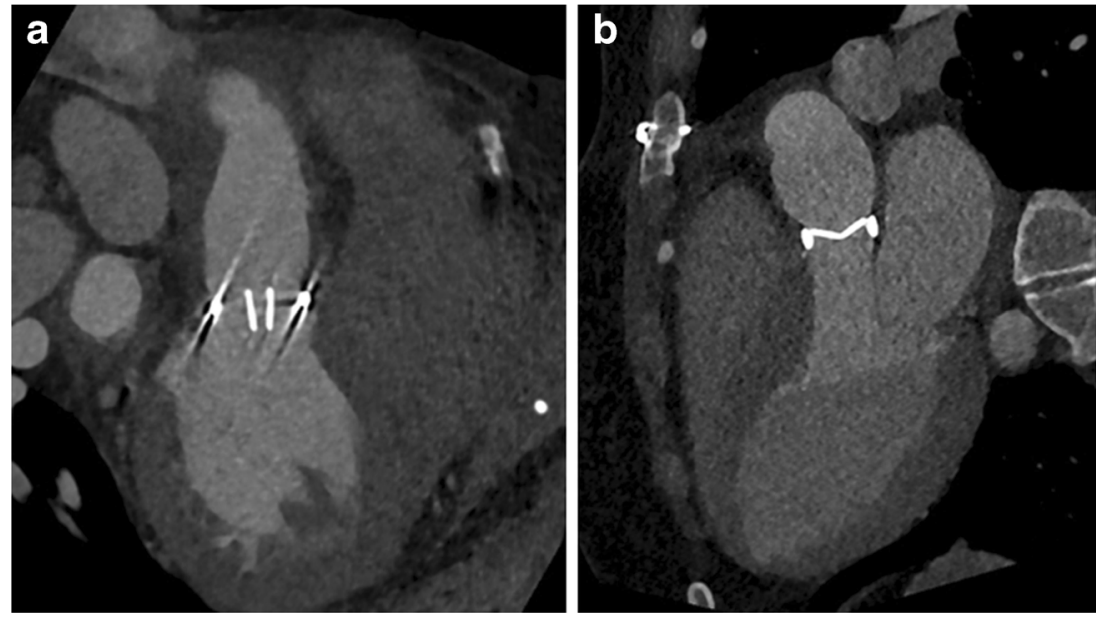

0.80. This means there was a good interobserver agreement for the non-enhanced scan and a good-to-excellent agreement for the other acquisitions and for scoring image quality of leaflet motion.

\section{Discussion}

Several studies have demonstrated the potential and incremental value of MDCT in patients with suspected PHV dysfunction [6-8]. However, these studies used retrospective ECG-gating CT angiography with a relatively high mean radiation dose of well over $10 \mathrm{mSv}$ for a single arterial phase acquisition only. Utilizing the capabilities of a modern dual-source scanner, we devised a comprehensive multiphase acquisition protocol for PHV assessment that aims to reduce radiation dose but at the same time increase coverage and assessment potential in multiple phases. Our study shows that this acquisition protocol was associated with good overall image quality and a moderate radiation dose of $8.3 \pm 3.0 \mathrm{mSv}$ while making three consecutive acquisitions. Furthermore, leaflet motion, which is an essential part of PHV evaluation with CT, could be visualized using the cine mode with a mean image quality of $3.9 \pm 1.4$. The use of three subsequent acquisitions offers a wide range of diagnostic possibilities and also provides essential information for planning possible re-interventions. Although the additional acquisition phases may not necessarily change the diagnosis per se, they may be helpful in interpretation and increase confidence. This, however, is difficult to quantify and therefore not included quantitatively in this study. The first, non-enhanced, scan shows the presence and extent of (peri)valvular calcifications. In case of endocarditis, calcifications in pseudoaneurysms around the aortic root allow discrimination between a chronic instead of an active endocarditis, as was the case in one of our patients. Moreover, this non-enhanced scan can identify sutures with polytetrafluorethylene felt pledgets, which were used in eight (19\%) of our patients. These pledgets can be isodense to contrast and mimic paravalvular leakage on CTA, thus a nonenhanced scan can be helpful to differentiate between the two (Fig. 6) [12]. The goal of the second CTA acquisition is to evaluate valve position and valve dynamics as well as to look for possible leakage, obstruction, pannus tissue, thrombus or
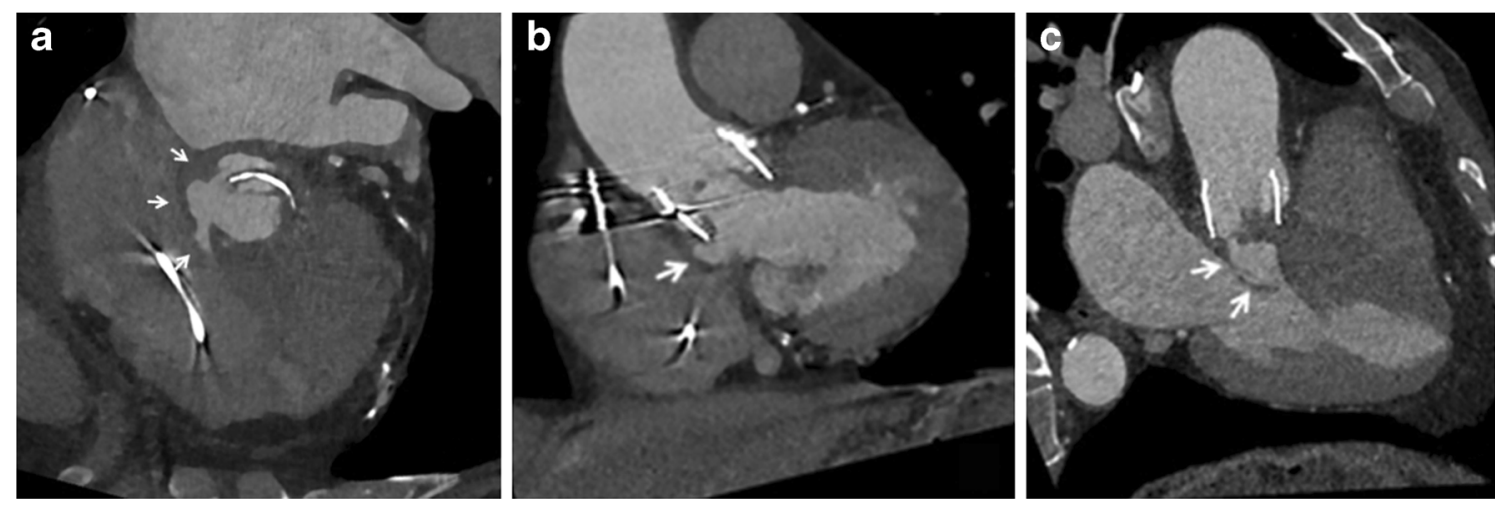

Fig. 5 Patient with aortic prosthetic heart valve (PHV) (TAVR, type Lotus valve) with suspected endocarditis. CT shows PVL and false aneurysm formation. A: Axial contrast-enhanced ECG-triggered CT image shows paravalvular leakage (arrows). B (coronal) and C (sagittal) contrast-enhanced ECG-triggered CT images nicely show the PHV in the aortic position and the false aneurysm formation underneath (arrows). 
Fig. 6 The non-contrast scan can identify sutures with polytetrafluorethylene felt pledgets. These pledgets can be isodense to contrast and mimic paravalvular leakage (PVL) on CT angiography (CTA) (A) and a non-contrast scan (B) can thus be helpful to differentiate between the two. Arrow indicates possible PVL on the CTA and confirms the hyperdense nature of the pledget on the non-contrast scan
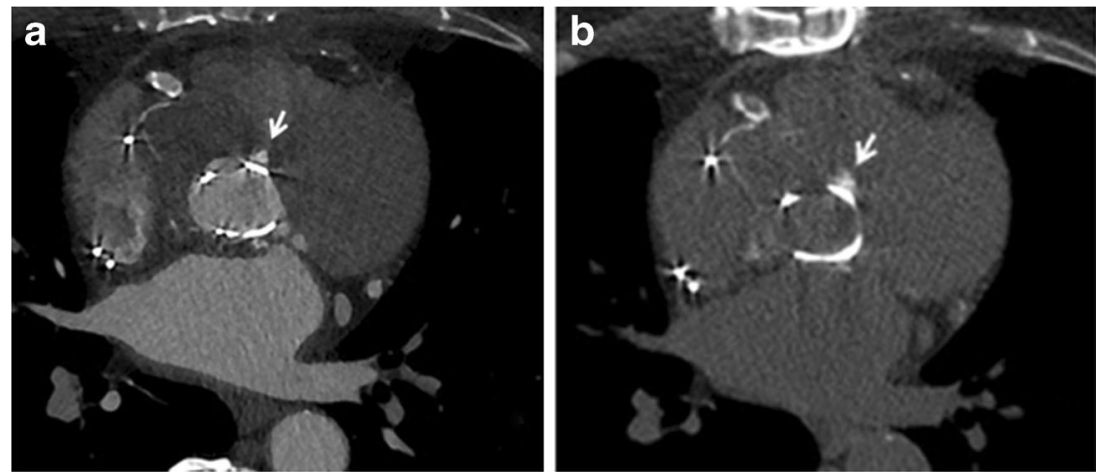

signs of endocarditis. We used prospective ECG-triggering with three stacks for our CTA acquisition, which lowered the dose by more than $20 \%$ in comparison to retrospective ECGtriggering at the cost of a short lack of data in the first $100 \mathrm{~ms}$ after the R-peak of the cardiac cycle [6-8]. By setting the scan length to exactly $14 \mathrm{~cm}$, three stacks were obtained with maximum individual stack coverage to ensure images were without stack artefacts at the level of the valve, as shown by our results (only two of 44 CTAs had stack artefacts, none of them affecting the valve). One might consider scanning a single stack only for the valve. However, the advantage of using three stacks is that the coronary arteries are completely visualized, which may allow an additional coronary angiography to be omitted in case of re-operation. Furthermore, it gives a good overview of the left ventricular outflow tract. In this acquisition, we used an ECG-trigger based on absolute millisecond (ms) time instead of a relative (\%) RR-trigger. A 'scan-on-scanoff' padding setting from $0-1,500 \mathrm{~ms}$ with $50-500 \mathrm{~ms}$ ECGpulsing resulted in a scan acquisition window that was guaranteed to provide the fastest onset of radiation after an R-peak (minimum delay is $\pm 100 \mathrm{~ms}$, which is $10 \%$ at $60 \mathrm{bpm}$ ). Radiation was maintained for $1,500 \mathrm{~ms}$ (which is a full heart cycle at $40 \mathrm{bpm}$ ) or cut off when the next R-peak occurred. This meant that regardless of the heart rate, a full heart cycle (starting from $100 \mathrm{~ms}$ ) was guaranteed, which makes this protocol very robust and easy to perform. The higher dose at 50-500 ms guaranteed high quality systolic images on which pannus, thrombus and leakage can be evaluated. In diastole, dose was
Fig. 7 Advantage of a delayed acquisition: differentiating thrombus from flow artefacts. One of the advantages of the delayed scan acquisition is the ability to better depict thrombus and differentiate thrombus from flow artefacts, since patients with a prosthetic heart valve (PHV) often have arrhythmias. In the first patient (A-B), the arterial C T angiography (CTA) acquisition (A) depicts a filling defect in the left atrial appendage (arrow). The delayed scan (B) shows that it involved a flow artefact with good filling of the left atrial appendage in this phase (arrows). Thrombus could thus be excluded. In the second patient (C-D), the arterial CTA acquisition $(\mathbf{C})$ also depicts a filling defect in the left atrial appendage (arrow). However, in this case, the delayed scan (D) confirms the presence of a thrombus (arrow)
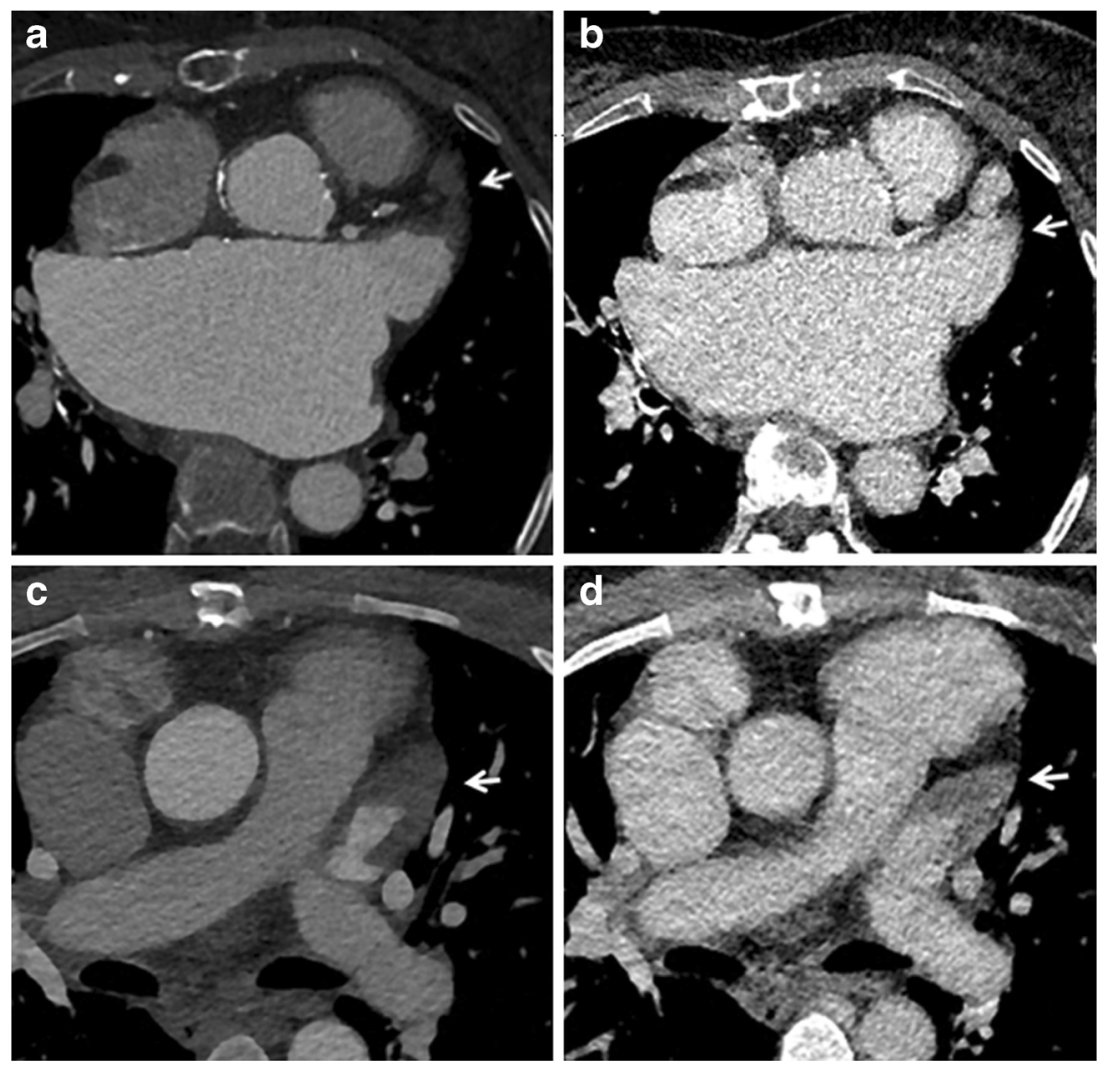
reduced to $20 \%$ (with higher noise levels), which is tolerable since this phase is only needed for evaluation of the leaflet motion and confirmation of pathology detected in systole. Moreover, prospective triggering reduced prosthetic heart valve-induced artefacts in comparison with retrospective ECG-gating [9]. Further development of this protocol could include automatic $\mathrm{kV}$ selection for this CTA acquisition in patients with a PHV with a limited amount of metallic components to further lower radiation dose.

The delayed scan comprised the entire chest and thus the full thoracic aorta, at a relatively low radiation cost. The advantages of this 'add-on' scan may be the identification of the extent of disease beyond valves, emphasizing infectious attenuation, better depicting thrombus and differentiating thrombus from flow artefacts (e.g. in the left atrial appendage, since patients with a PHV often have arrhythmias). In five patients (12\%) we could thus differentiate between thrombus and flow artefacts (Fig. 7). Besides optimization of CT acquisition parameters, another way to achieve dose reduction is to optimize CT image reconstruction through iterative reconstruction techniques. This alternative image reconstruction method allows imaging at a lower radiation-dose with similar noise levels and image quality compared to routine dose filtered backprojection (FBP), thus reducing dose without compromising image quality [13].

Although a mean radiation dose of $8.3 \pm 3.0 \mathrm{mSv}$ for all three acquisitions combined is considerably lower than reported previously [6-8], it is not minimal. However, one must take into account that this patient population was relatively old and represents a population that often has serious pathology with relatively high morbidity and mortality, e.g. due to endocarditis, with a genuine possibility of needing a highrisk reoperation. This imposes a good balance between lowering the radiation dose as much as possible but still gathering all the information needed for good clinical decision making and preoperative planning. In follow-up CT examinations, and depending on the clinical question, the non-enhanced or delayed scan can be omitted and the CTA can be obtained using only one stack through the valve, where relevant. As underlined in several recent studies, another additional benefit of $\mathrm{CT}$ is the power to detect leaflet thickening (most likely due to thrombus formation) of biological and transcatheter PHVs that was not detected by TTE [14-16]. The possible addition of CT in the routine work-up early after PHV implantation also necessitates lowering the radiation dose as much as possible.

Our study had some limitations. In this descriptive study of a new CT protocol for the evaluation of PHVs, no comparison with other acquisition protocols in the same or a similar group of patients was made. Furthermore, albeit irrelevant to our main outcome of image quality, no comparison with a reference standard was made regarding pathological findings.

\section{Conclusion}

A novel comprehensive CT image acquisition protocol is presented that allows for both dynamic reconstructions to assess PHV leaflet motion and high-resolution anatomical images with good image quality, using third-generation dual-source $\mathrm{CT}$ and iterative reconstruction techniques. The moderate radiation dose of $8.3 \pm 3.0 \mathrm{mSv}$ for this three-phase acquisition is - albeit not minimal - substantially lower (> $20 \%$ reduction) than previous (usually retrospectively ECG-gated) singlephase image acquisition techniques and can be considered acceptable when seen in relation to the pathology, the risks and possible re-intervention in this patient population.

Funding The authors state that this work has not received any funding.

\section{Compliance with ethical standards}

Guarantor The scientific guarantor of this publication is Ricardo Budde, Erasmus MC, Rotterdam.

Conflict of interest The authors of this manuscript declare no relationships with any companies whose products or services may be related to the subject matter of the article.

Statistics and biometry No complex statistical methods were necessary for this paper.

Informed consent Written informed consent was waived by the Institutional Review Board.

Ethical approval Institutional Review Board approval was not required because the acquisition was part of the routine clinical workup, and data were gathered retrospectively. No additional acquisitions were made specifically for this study. Patient data were retrieved from the electronic patient files. A waiver for retrospective evaluation of the data was obtained from the medical ethics committee.

\section{Methodology \\ - retrospective \\ - observational \\ - performed at one institution}

Open Access This article is distributed under the terms of the Creative Commons Attribution 4.0 International License (http:// creativecommons.org/licenses/by/4.0/), which permits unrestricted use, distribution, and reproduction in any medium, provided you give appropriate credit to the original author(s) and the source, provide a link to the Creative Commons license, and indicate if changes were made.

\section{References}

1. Habets J et al (2011) Diagnostic evaluation of left-sided prosthetic heart valve dysfunction. Nat Rev Cardiol 8:466-478

2. Lancellotti $P$ et al (2016) The clinical use of stress echocardiography in non-ischaemic heart disease: recommendations from the European Association of Cardiovascular Imaging and the American Society of Echocardiography. Eur Heart J Cardiovasc Imaging 11:1191-1229 
3. Sucha D et al. (2015) Multimodality imaging assessment of prosthetic heart valves. Circ Cardiovasc Imaging 8(9)

4. Habets J et al (2011) Prosthetic heart valve assessment with multidetector-row CT: imaging characteristics of 91 valves in 83 patients. Eur Radiol 21:1390-1396

5. Ghersin E et al (2013) ECG-gated MDCT after aortic and mitral valve surgery. AJR 203:596-604

6. Sucha D et al (2016) Diagnostic evaluation and treatment strategy in patients with suspected prosthetic heart valve dysfunction: The incremental value of MDCT. J Cardiovasc Comput Tomogr 5:398-406

7. Habets $\mathrm{J}$ et al (2014) Cardiac computed tomography angiography results in diagnostic and therapeutic change in prosthetic heart valve endocarditis. Int J Card Imaging 30:377-387

8. Chenot $\mathrm{F}$ et al (2010) Evaluation of anatomic valve opening and leaflet morphology in aortic valve bioprosthesis by using multidetetor CT: comparison with transthoracic echocardiography. Radiology 255:377-385

9. Symersky P et al (2012) Prospective ECG triggering reduces prosthetic heart valve-induced artefacts compared with retrospective ECG gating on 256-slice CT. Eur Radiol 22:1271-1277
10. Deak PD, Smal Y, Kalender WA (2010) Multisection CT protocols: sex- and age-specific conversion factors used to determine effective dose from dose-length product. Radiology 257(1):158-166

11. Symersky P, Budde RPJ, Prokop M, de Mol BAJM (2011) Multidetector-Row Computed Tomography Imaging Characteristics of Mechanical Prosthetic Valves. J Heart Valve Dis 20(2):216-222

12. Habets $J$ et al (2012) CT attenuation measurements are valuable to discriminate pledgets used in prothetic heart valve implantation from paravalvular leakage. Br J Radiol:616-621

13. Willemink MJ et al (2013 Jun) Iterative reconstruction techniques for computed tomography Part 1: technical principles. Eur Radiol 23(6):1623-1631

14. Makkar RR, Fontana G, Jilaihawi H, et al. (2015) Possible Subclinical Leaflet Thrombosis in Bioprosthetic Aortic Valves. N Engl J Med

15. Leetmaa T, Hansson NC, Leipsic J et al (2015) Early Aortic Transcatheter Heart Valve Thrombosis. Circ Cardiovasc Interv 8:1-9

16. Pache G, Schoechlin S, Blanke P et al (2016) Early hypo-attenuated leaflet thickening in balloon-expandable transcatheter aortic heart valves. Eur Heart J 37:2263-2271 